\title{
Tracheostomy in patients with COVID-19-related acute respiratory distress syndrome - Characteristics, complications, and a comparison between early and late tracheostomy: A retrospective observational study
}

\section{Anna Hansson ( $\square$ annha665@student.liu.se )}

Linköping University: Linkopings universitet https://orcid.org/0000-0002-2645-3484

\section{Ola Sunnergren}

Department of otorhinolaryngology, Region Jönköping County, Sweden

\section{Anneli Hammarskjöld}

Department of anaesthesiology and intensive care medicine, Ryhov county hospital, Sweden

\section{Catarina Alkemark}

Department of anaesthesiology and intensive care medicine, Ryhov county hospital, Sweden

\section{Knut Taxbro}

University Hospital Münster Department of Anesthesiology and Intensive Care Medicine, Ryhov county hospital, Sweden

\section{Research}

Keywords: COVID-19, tracheostomy, acute respiratory distress syndrome, ICU

Posted Date: May 24th, 2021

DOI: https://doi.org/10.21203/rs.3.rs-543176/v1

License: (c) (1) This work is licensed under a Creative Commons Attribution 4.0 International License. Read Full License

Version of Record: A version of this preprint was published at Health Science Reports on April 22nd, 2022. See the published version at https://doi.org/10.1002/hsr2.595. 


\section{Abstract}

Background As the coronavirus disease (COVID-19) pandemic spread worldwide in 2020, the number of patients requiring intensive care and invasive mechanical ventilation (IMV) has increased rapidly. Tracheostomy has several advantages over oral intubation in critically ill patients, including the facilitation of prolonged mechanical ventilation. However, the optimal timing of the procedure remains unclear. During the pandemic, early recommendations suggested that tracheostomy should be postponed, as the potential benefits were not certain to exceed the risk of viral transmission to healthcare workers. The aim of this study was to assess the utility of tracheostomy in patients with COVID-19-related acute respiratory distress syndrome, in terms of patient and clinical characteristics, outcomes, and complications, by comparing between early and late tracheostomy.

Methods A multicentre, retrospective observational study was conducted in Jönköping County, Sweden. Between 14 March 2020 and 13 March 2021, 117 patients were included in the study. All patients $\geq 18$ years of age with confirmed COVID-19 who underwent tracheostomy were divided into two groups based on the timing of the procedure ( $\leq />7$ days). Outcomes including the time on IMV, intensive care unit (ICU) length of stay, and mortality 30 days after ICU admission, as well as complications due to tracheostomy were compared between the groups.

Results Early tracheostomy ( $<7$ days, $n=56$ ) was associated with a shorter duration of mechanical ventilation (7 [Inter Quartile Range, IQR 12], p = 0.001) as well as a shorter ICU stay (8 [IQR 14], p = 0.001). The mortality rates were equal between the groups. The most frequent complication of tracheostomy was minor bleeding. With the exception of a higher rate of obesity in the group receiving late tracheostomy, the patient characteristics were similar between the groups.

Conclusions This study showed that early tracheostomy was safe and associated with a shorter time on IMV as well as a shorter ICU length of stay, implicating possible clinical benefits in critically ill COVID-19 patients. However, it is necessary to verify these findings in a randomised controlled trial.

Trial Registration: Not required

\section{Background}

In December 2019, an unknown form of pneumonia was reported in Wuhan, China(1). Within the first weeks of 2020, the coronavirus disease (COVID-19) had spread worldwide, causing a pandemic(2). In May 2021, the World Health Organization reported over 152 million cases and three million deaths globally due to COVID-19(3). In Sweden, the number of cases peaked in April 2020 and then again in December 2020, creating the characteristic "first and second wave".

As the virus spread, the number of patients requiring admission to the intensive care unit (ICU) increased rapidly, leading to massive challenges for the health care system(4) in general and ICUs in particular. COVID-19 causes critical illness in $5 \%$ of cases $(5,6)$ and one of the most common and life-threatening 
complications of COVID-19 is acute respiratory distress syndrome (ARDS)(7). ARDS is characterised by hypoxemic respiratory failure, pulmonary epithelial injury in the alveoli, and a systemic inflammatory response(8). Patients with ARDS due to COVID-19 generally require prolonged invasive mechanical ventilation (IMV) and are, therefore, often considered for tracheostomy(4).

The predominant indication for tracheostomy in patients with ARDS due to COVID-19 is to facilitate prolonged IMV and aid when difficult weaning is anticipated(9). Tracheostomy is associated with a lower requirement for sedatives and improved patient comfort, as well as optimised clearance of airway secretions. It facilitates oral care and reduces the risk of ventilator-associated pneumonia and aspiration during weaning, while avoiding complications of long-term oral intubation, such as tracheal stenosis(10, 11). In addition, tracheostomy tubes provide less need for breathing by bypassing the mouth and pharynx, thus creating less airway dead space(4). Since patients can be discharged to intermediate care facilities before decannulation, tracheostomy could potentially decrease the time in the ICU(12) liberating much needed resources.

Tracheostomy can be performed using either open surgical or percutaneous techniques. Studies have not shown significant differences in outcomes between the methods used for tracheostomy in COVID-19 patients(13).

Performing tracheostomy has several advantages over oral intubation in critically ill patients requiring mechanical ventilation(14). However, the optimal timing of the procedure has not been determined. Data on "early" vs "late" tracheostomy regarding outcomes such as mortality and length of ICU and hospital stay are conflicting, and no consensus has yet been reached(15). As the clinical trajectory of COVID-19 was unclear at the onset of the pandemic, the timing of tracheostomy was even more ambiguous in these patients(16).

Since tracheostomy is an aerosol-generating procedure(17), it is considered to be associated with a high risk of viral transmission to healthcare personnel, based on experience from the severe acute respiratory syndrome epidemic in 2003(18). Because of this, recommendations about being restrictive when performing tracheostomy in COVID-19 patients emerged at the onset of the pandemic $(19,20)$. Experts in otolaryngology suggested that tracheostomy should be delayed when the patient is cleared of infection, unless an adequate airway cannot be maintained with oral intubation(21). In June 2020, the American College of Chest physicians recommended that tracheostomy should be considered in COVID-19 patients, although evidence on the optimal timing of the procedure is still lacking(22).

With limited data on this population, healthcare providers were unable to determine whether the potential benefits of tracheostomy in COVID-19 patients exceeded the risk of infection in healthcare workers. During the pandemic, liberating ICU resources in a time of scarcity and maintaining patient care at an optimum level was a delicate balancing act.

To date, there are limited data on the clinical course and outcomes of tracheostomy in patients with COVID-19. In addition, little is known regarding the optimal timing of tracheostomy in these patients. 
These clinical questions occur daily when treating patients with COVID-19; however, the evidence for providing answers is limited. The aim of this study was to compare early and late tracheostomy in patients with COVID-19-related ARDS with regard to the clinical course and rates of tracheostomy-related complications. We assessed past recommendations regarding the timing of the procedure and the potential utility of early tracheostomy in patients with COVID-19.

\section{Methods}

This was a multicentre, retrospective observational study conducted at three non-academic rural hospitals in Jönköping County in southern Sweden.

\section{Patient selection and methods}

The study included all adult patients ( $\geq 18$ years old) who were admitted to the ICU in Jönköping County between 14 March 2020 and 13 March, 2021 (Fig. 1). All included patients were diagnosed with severe acute respiratory syndrome coronavirus 2 (SARS-CoV-2) infection, as confirmed by positive polymerase chain reaction results using nasopharyngeal swabs. The final date for data cut-off was 14 April 2021. Patients who were treated in the ICU in Jönköping County but underwent tracheostomy elsewhere were excluded. For patients transferred from our hospitals to different regions after receiving tracheostomy, data were collected only for the time in Jönköping County.

Patients who underwent tracheostomy were categorised into two groups based on the timing of the procedure. Those who were on mechanical ventilation for $\leq 7$ days prior to surgery were included in the "early" group and those who were on mechanical ventilation for $>7$ days were included in the "late" group.

Medical records from Cosmic $^{\text {TM }}$ and MetaVision ${ }^{T M}$ were reviewed retrospectively by trained abstractors to ensure accuracy.

\section{Main outcomes and measures}

Patient demographics included age, sex, body mass index (BMI), presence of comorbidities (cardiac disease, hypertension, diabetes, kidney/liver/lung disease, immunosuppression, neuromuscular disease, and active or previous cancer), as well as present or previous smoking habits and blood group. The primary outcome was the duration of IMV. The secondary outcomes included number of days on IMV prior to tracheostomy, time of decannulation, number of hours on mechanical ventilation in the prone position, ICU length of stay, all-cause mortality (within 30 days of ICU admission), and complications associated with tracheostomy (haemorrhage, aspiration, displaced tracheal cannula, tracheal injury, failed surgery, accidental decannulation, stoma infection, perioperative hypoxemia, pneumothorax/pneumomediastinum/subcutaneous emphysema, fistulas, and airway obstruction related to tracheostomy, and procedure-related death)

\section{Definitions}


Co-morbidities were defined by ICD-10 codes in the medical records, that is, cardiovascular disease (I25 and/or I50), hypertension (I10), diabetes (E10/E11), asthma (J45), and chronic obstructive pulmonary disease (J44) in combination with active treatment. Immunosuppression was classified as systemic steroid treatment, radiation, or chemotherapy $<6$ months prior to hospital admission(23). Kidney function was defined using the Kidney Disease: Improving Global Outcomes classification of chronic kidney disease based on the glomerular filtration rate (GFR) $\mathrm{mL} / \mathrm{min} / 1.73 \mathrm{~m}^{2}$.

Complications associated with tracheostomy were defined as events that had a potential negative impact on the patients' clinical course and thus required intervention. All events occurring from the time of the procedure to the time of decannulation were included. The definitions of the complications are outlined in Table 1. 
Table 1 - Definitions of tracheostomy-related complications

Haemorrhage

In tracheal stoma, needing manual or pharmacological intervention

Displaced tracheal cannula

Displacement requiring manual repositioning

Aspiration

Visualization of gastric contents in

the airway or clinical indications of aspiration

Tracheal injury

Failed surgery
Lacerations or wounds related to the procedure or from chafing by the tracheal cannula

Discontinuation of the procedure due to complications or unforeseen events

\begin{tabular}{ll}
$\begin{array}{l}\text { Pneumothorax/pneumomediastinum/subcutaneous } \\
\text { emphysema }\end{array}$ & $\begin{array}{l}\text { Verified by chest CT or clinical } \\
\text { examination }\end{array}$ \\
\hline Airway obstruction & $\begin{array}{l}\text { Causing severe dyspnoea, needing } \\
\text { physician attention }\end{array}$ \\
\hline Accidental decannulation & Tracheal cannula dislodgement \\
\hline Stoma infection & Culture verified infection resulting \\
& in change in antibiotics \\
\hline Fistula & Tracheoarterial or \\
& tracheoesophageal fistula \\
\hline
\end{tabular}

\section{Ethical considerations}

This study was approved by the Swedish Ethical Review Authority on August 26, 2020 (Dnr 2020-02758). Patient consent was waived due to the retrospective nature of the study.

Statistical analysis 
All statistical analyses were performed using IBM SPSS Statistics (version 27.0; IBM Corp., Armonk, NY, USA). Data are presented as descriptive statistics, with categorical variables reported as frequencies (percentages). The continuous variables are presented as medians (interquartile ranges [IQRs]). The differences between the groups were tested using the chi-square test, the Mann-Whitney U test, Fisher's exact test, or Student's t-test, as appropriate. All tests were two-sided, and statistical significance was set at $p<0.05$.

\section{Results}

\section{Patient characteristics}

Between 14 March 2020 and 13 March 2021, 264 patients with COVID-19 were admitted to the ICU in three hospitals in Jönköping County, Sweden. Of the 204 patients requiring IMV, 117 (57\%) underwent tracheostomy (fig. 1). The baseline characteristics are shown in Table 2. The baseline characteristics were similar between the early and late tracheostomy groups, with the exception that

immunosuppressant treatment prior to ICU admission was more common in the late tracheostomy group $(p=0.026)$. In both the groups, the patients were predominantly men, with a median age of 66 years. Hypertension was the most frequent comorbidity (55\%), followed by type 2 diabetes (28\%). In total, $45 \%$ of the patients had a BMI of $\geq 30$, with a significantly higher BMI $(p=0.025)$ among patients in the late tracheostomy group. All tracheostomies were performed at the bedside in the ICU. Open surgical tracheostomy was performed in $116(99.1 \%)$ cases.

One patient remained on mechanical ventilation at the time of the final data cut-off. Six patients had no documented date of decannulation. 
Table 2 - Baseline demographic data prior to initial ICU admission 
Early tracheostomy Late tracheostomy Total

$(\mathrm{n}=56) \quad(\mathrm{n}=61) \quad(\mathrm{n}=117)$

Age, median (min-max)

$67(22-87)$

$65(18-83) \quad 66(18-87)$

Gender, n (\%)

Male

$46(82)$

$44(72)$

90 (77)

Female

$10(18)$

$17(28)$

$27(23)$

Cardiovascular disease, $\mathrm{n}(\%)$

9 (16)

$15(25)$

$24(21)$

Hypertension, n (\%)

$27(48)$

$37(61)$

64 (55)

Diabetes, n (\%)

Type 1

$2(4)$

2 (3)

$4(3)$

Type 2

13 (23)

20 (33)

$33(28)$

Liver cirrhosis, n (\%)

$0(0)$

$1(2)$

1 (1)

Neuromuscular disease, n (\%)

$3(6)$

2 (3)

$5(4)$

Immunosuppression, n (\%)

3 (6)

$12(20)$

15 (13)

Asthma, n (\%)

$6(11)$

8 (13)

$14(12)$

COPD, n (\%)

$6(11)$

5 (8)

11 (9)

Other pulmonary disease, $\mathrm{n}(\%)$

7 (13)

9 (15)

$16(14)$

Cancer, n (\%)

Active

$4(7)$

$1(2)$

$5(4)$

Previous

$5(9)$

$1(2)$

$6(5)$

Kidney function, n (\%)

$22(36)$

$49(42)$

KDIGO 1

$27(48)$

37 (60)

66 (56)

KDIGO 2

$29(52)$

$0(0)$

$0(0)$

KDIGO 3

$0(0)$

2 (3)

2 (2)

KDIGO 4

$0(0)$

$0(0)$

$0(0)$

KDIGO 5

$0(0)$

Blood type, n (\%) 

0
19 (34)
$25(41)$
44 (38)
A
$29(52)$
24 (39)
$53(45)$
$B$
$2(4)$
11 (18)
$13(11)$
$A B$
$3(6)$
$1(2)$
4 (3)

Active/previous smoking, n (\%)

\begin{tabular}{lccc} 
& $19(34)$ & $21(34)$ & $40(34)$ \\
\hline $\mathrm{BMI} \mathrm{kg} / \mathrm{m}^{2}$ median $[\mathrm{IQR}]$ & $28[26-33]$ & $31[28-35]$ & $30[27-34]$ \\
\hline $\mathrm{BMI} \geq 30 \mathrm{~kg} / \mathrm{m}^{2}, \mathrm{n}(\%)$ & $21(38)$ & $32(53)$ & $53(45)$ \\
\hline $\mathrm{SAPS} 3$, median $[\mathrm{IQR}]$ & $60[49-63]$ & $58[52-63]$ & $59[51-63]$ \\
\hline High-dose prophylactic LMWH (\%) & $48(86)$ & $38(62)$ & $86(74)$
\end{tabular}

Clinical characteristics of the study population, in total and divided by early and late tracheotomy. Categorical variables are reported as frequencies (\%) and continuous variables as medians [IQR]

COPD = chronic obstructive pulmonary disease; SAPS3 = simplified acute physiology score 3; High-dose $L M W H=\geq 9000$ units/day low molecular weight heparin

\section{Tracheostomy outcomes}

Compared with those who received late tracheostomy, patients who received early tracheostomy had a shorter median ICU stay, with an absolute difference of 8 days $(p=0.002)$. Early tracheostomy was also associated with fewer days of IMV ( $p=0.001)$ than those in late tracheostomy (Table 3$)$. There was no difference in the time from tracheostomy to weaning from mechanical ventilation or time of decannulation. In both the early and late tracheostomy groups, there were six deaths $(10 \%$ and $11 \%$, respectively; $p=1)$. The median durations from intubation to tracheostomy were 5 and 10 days in the early and late tracheostomy groups, respectively.

Table 3 - Outcomes 


\begin{tabular}{|c|c|c|c|}
\hline & $\begin{array}{c}\text { Early } \\
\text { tracheotomy } \\
\text { (<7 days) }\end{array}$ & $\begin{array}{c}\text { Late } \\
\text { tracheotomy } \\
\text { (>7 days) }\end{array}$ & $\begin{array}{c}\mathrm{p}- \\
\text { value }\end{array}$ \\
\hline Days on ventilator [IQR] & $13[9-20]$ & $20[16-28]$ & 0.001 \\
\hline Total ICU stay [IQR] & $16[12-26]$ & $24[19-34]$ & 0.002 \\
\hline Days from tracheotomy to decannulation [IQR] & $13[7-23]$ & $14[8-22]$ & 0.914 \\
\hline Days in IMV at tracheotomy, median [IQR] & $5[4-6]$ & $10[9-11]$ & \\
\hline Hours of IMV in prone position, median [IQR] & $32[15-54]$ & 56 [21-99] & 0.052 \\
\hline All-cause mortality, 30 days, $\mathrm{n}(\%)$ & $6(11)$ & $6(10)$ & 1 \\
\hline $\begin{array}{l}\text { Discharged to intermediate care prior to } \\
\text { decannulation, } \mathrm{n}(\%)\end{array}$ & $30(54)$ & $29(48)$ & 0.46 \\
\hline \multicolumn{4}{|c|}{ Clinical outcomes compared between early and late tracheostomy. Categorical variables are } \\
\hline
\end{tabular}

Complications due to tracheostomy occurred in 42 patients (36\%). The most frequent complication was stoma haemorrhage (requiring manual or pharmacological intervention), which occurred in 26 (22\%) patients. The rate of complications was higher in the group receiving late tracheostomy; however, there were no differences in terms of each complication by itself. A summary of the recorded complications is presented in Table 4.

Table 4 - Complications 


\section{( $<7$ days) $\quad$ ( $>7$ days)}

Haemorrhage

Perioperative

$0(0)$

2(3)

0.5

Early ( $\leq 2$ days)

6(11)

$8(13)$

0.78

Late ( $>2$ days)

\section{Aspiration}

Perioperative

$0(0)$

$0(0)$

Early ( $\leq 2$ days)

$0(0)$

$0(0)$

Late ( $>2$ days)

$0(0)$

$1(2)$

0.92

Tracheal injury

$1(2)$

$0(0)$

0.67

Failed surgery

$1(2)$

$0(0)$

0.48

Accidental decannulation

2(4)

$1(2)$

0.48

Perioperative hypoxaemia, $\mathrm{SpO}_{2}<80 \%$

6(11)

$9(15)$

0.59

Culture verified stoma infection

$1(2)$

$9(16)$

Pneumothorax/

pneumomediastinum/

sc emphysema

\begin{tabular}{lccc}
\hline Fistula & $0(0)$ & $0(0)$ & -- \\
\hline Airway obstruction & $2(4)$ & $6(10)$ & 0.28 \\
\hline Procedure related death & $0(0)$ & $0(0)$ & -- \\
\hline Any complication & $19(34)$ & $23(38)$ & 0.014
\end{tabular}


Complications of tracheotomy, compared between early and late tracheotomy. Categorical variables are reported as frequencies and percentages, $n(\%)$.

Sc $=$ subcutaneous

\section{Discussion}

Our results suggest that early tracheostomy ( $\leq 7$ days) is associated with a shorter length of stay in the ICU and fewer days on IMV than those in late tracheostomy (> 7 days). These findings illustrate the potential clinical benefits, including easier weaning from mechanical ventilation and performing tracheostomy earlier than what was recommended by international guidelines in the initial phase of the pandemic. Our results are in agreement with those of other studies $(16,24)$. However, the selection bias in the late tracheostomy group should be considered. Patients in the late group possibly had increased complexity in their clinical course compared to that in patients in the early group. This was because patients in the late tracheostomy group had a higher incidence of immunosuppressant treatment (deduced from the medical history) and required a longer time in the prone position compared to the patients in the early tracheostomy group. The median time in the prone position during ICU stay was $32 \mathrm{~h}$ in the early tracheostomy group and $56 \mathrm{~h}$ in the late tracheostomy group.

The BMI was significantly higher in patients who received late tracheostomy than in those who received early tracheostomy, with $53 \%$ of patients being obese $(\mathrm{BMI} \geq 30)$ in the late group. As obesity is a risk factor for increased severity of COVID-19(25), this may have impacted the timing of tracheostomy being considered safe and profitable for the patient. Prone positioning has been shown to improve outcomes in patients with severe ARDS(26) and it may be more difficult to manage tracheostomy in obese patients while in the prone position. Hence, obesity may have influenced decisions to postpone tracheostomy with the ambition to successfully extubate the patient or until prone positioning was no longer needed. In addition, open tracheostomy on obese, critically ill patients is associated with an increased risk of severe complications(27).

Comparison of all-cause mortality 30 days after initial ICU admission showed no advantages for early or late tracheostomy. These findings are similar to those of other studies, including the landmark TracMan trial $(28,29)$. However, the low overall mortality rate in this study compared to that in other studies on tracheostomy in COVID-19 patients limits comparativeness, as a small sample may influence statistical analysis.

In this study, clinically relevant complications due to tracheostomy occurred in 42 (36\%) patients, compared to complication rates of $4 \%-22 \%$ reported in other studies $(4,28,30,31)$. Although the total complication rates in this study somewhat exceed those in similar studies, the rates of each complication by itself are comparable to those of other studies. This indicates a variation in the definition of 
complications between studies, making comparison difficult. The difficulty in comparison is further aggravated by divergence in the tracheostomy technique used due to local traditions and preferences.

The most frequent complication in our patient group was haemorrhage in the tracheal stoma, appearing in 26 cases (22\%). This was expected, as haemorrhage is reported to be one of the most common complications of tracheostomy in several studies. Due to the thrombogenic nature of COVID-19, higher than usual doses of prophylactic low-molecular-weight heparin (LMWH) are commonly used. Although not statistically significant, 21 (81\%) patients who received high-dose LMWH had a stomal haemorrhage compared to $5(19 \%)$ among those who received low-dose LMWH $(p=0.45)$. No major bleeding due to tracheostomy was observed. Complications, when assessed as composite variable, occurred more frequently in the group receiving late tracheostomy. However, when the specific complications were compared separately, no significant differences were observed between the groups (Table 4).

The decision to perform tracheostomy was made at the discretion of the consultant intensive care physician at each hospital. As ICU beds rapidly filled up and the supply of sedatives declined, the threshold for performing early tracheostomy was low. This approach was retained during the "second wave" of the pandemic, and there was no difference in the time until tracheostomy between the first and second waves. The procedure was performed in collaboration among anaesthesiologists, otolaryngologists, and general surgeons. All but one tracheostomy procedure was performed with an open method, as this technique could allocate workload among specialties. Guidelines published in May 2020 also suggested that open tracheostomy generated less aerosols compared to percutaneous tracheostomy, thereby reducing the potential risk of viral transmission to healthcare workers(32).

There was no difference between the groups in the time from tracheostomy to liberation from mechanical ventilation, suggesting that the reduction in ventilator time is dependent on the shortening time from intubation to tracheostomy. These results favour an earlier approach, confirming the findings of similar studies. On the contrary, others continue to recommend a more conservative approach, which includes waiting for as long as 21 days before performing tracheostomy in COVID-19 patients(33, 34). With no clear consensus, the decision regarding patient selection and timing of tracheostomy should be carefully evaluated and should not be made merely based on the time elapsed since intubation. As the course of this disease progresses, we will continue to learn more about the treatment strategies.

This study had several limitations. First, due to its retrospective nature and lack of randomisation between early and late tracheostomy, the risk of bias was high. Second, one patient remained in the ICU, and six patients had not been decannulated at the time of data cut-off, introducing some uncertainty regarding the length of stay in the ICU, duration of mechanical ventilation, and number of days until decannulation. This study also had several strengths. We included all eligible patients during the first year of the pandemic in our region, and no patients were lost to follow-up. Data were collected by several trained abstractors, and several key variables were collected automatically and prospectively by the same patient data management system in all participating hospitals. 


\section{Conclusions}

This study showed that early tracheostomy is feasible and associated with shorter time on IMV and shorter ICU length of stay, indicating a possible clinical benefit of early tracheostomy in treating critically ill COVID-19 patients. However, our findings need to be verified through randomised controlled trials. There were no differences in tracheostomy-related complications between the early and late tracheostomy groups.

\section{List Of Abbreviations}

ARDS - Acute respiratory distress syndrome

$\mathrm{BMI}$ - Body mass index

COVID-19 - coronavirus disease

GFR - Glomerular filtration rate

ICU - Intensive care unit

IMV - Invasive mechanical ventilation

IQR - Interquartile range

PCR - Polymerase chain reaction

\section{Declarations}

\section{Ethics approval and consent to participate}

This study was approved by the Swedish Ethical Review Authority on August 26, 2020

(Dnr 2020-02758). Patient consent was waived due to the retrospective nature of the study.

\section{Consent for publication}

All authors gave consent for publication.

\section{Availability of data and materials}

The data that support the findings of this study are available from the corresponding author upon reasonable request.

\section{Competing interests}

None declared by any author. 


\section{Funding}

This trial was supported by Futurum, the Academy for Healthcare, Jönköping County Council, Jönköping, Sweden.

\section{Author's contributions}

Idea, ethics approval (KT, OS)

Creation of database $(\mathrm{KT}, \mathrm{AH})$

Data collection (AH, AHd, $\mathrm{CA})$

Writing draft manuscript (AH)

Statistical analysis $(\mathrm{AH}, \mathrm{KT})$

Intellectual contribution to manuscript and acceptance of the final version (All)

\section{Acknowledgement}

We would like to express our gratitude to the staff of Jönköping County involved in caring for the COVID19 patients. We would also like to acknowledge S. Wennerholm and R. Svensson for their meticulous work in collecting data.

\section{References}

1. Zhu J, Ji P, Pang J, Zhong Z, Li H, He C, et al. Clinical characteristics of 3062 COVID-19 patients: A meta-analysis. J Med Virol. 2020;92(10):1902-14.

2. Karadag E. Increase in COVID-19 cases and case-fatality and case-recovery rates in Europe: A crosstemporal meta-analysis. J Med Virol. 2020;92(9):1511-7.

3. Weekly operational update on COVID-19 World Health Organization2021 [Available from: https://www.who.int/publications/m/item/weekly-operational-update-on-covid-10-3-may-2021.

4. Tang Y, Wu Y, Zhu F, Yang X, Huang C, Hou G, et al. Tracheostomy in 80 COVID-19 Patients: A Multicenter, Retrospective, Observational Study. Front Med (Lausanne). 2020;7:615845.

5. Martínez-Téllez E, Orús Dotú C, Trujillo-Reyes JC, Guarino M, Cladellas Gutiérrez E, Planas Cánovas G, et al. Tracheotomy in patients COVID-19: A necessary high risk procedure. Two center experience. Arch Bronconeumol. 2020;56(10):673-4.

6. Tornari C, Surda P, Takhar A, Amin N, Dinham A, Harding R, et al. Tracheostomy, ventilatory wean, and decannulation in COVID-19 patients. Eur Arch Otorhinolaryngol. 2020:1-10.

7. Yang X, Yu Y, Xu J, Shu H, Xia J, Liu H, et al. Clinical course and outcomes of critically ill patients with SARS-CoV-2 pneumonia in Wuhan, China: a single-centered, retrospective, observational study. 
Lancet Respir Med. 2020;8(5):475-81.

8. Li X, Ma X. Acute respiratory failure in COVID-19: is it "typical" ARDS? Crit Care. 2020;24(1):198.

9. Courtney A, Lignos L, Ward PA, Vizcaychipi MP. Surgical Tracheostomy Outcomes in COVID-19Positive Patients. OTO Open. 2021;5(1):2473974x20984998.

10. Shiba T, Ghazizadeh S, Chhetri D, St John M, Long J. Tracheostomy Considerations during the COVID-19 Pandemic. OTO Open. 2020;4(2):2473974x20922528.

11. Nseir S, Di Pompeo C, Jozefowicz E, Cavestri B, Brisson H, Nyunga M, et al. Relationship between tracheotomy and ventilator-associated pneumonia: a case control study. Eur Respir J. 2007;30(2):314-20.

12. Mattioli F, Fermi M, Ghirelli M, Molteni G, Sgarbi N, Bertellini E, et al. Tracheostomy in the COVID-19 pandemic. Eur Arch Otorhinolaryngol. 2020;277(7):2133-5.

13. Long SM, Chern A, Feit NZ, Chung S, Ramaswamy AT, Li C, et al. Percutaneous and Open Tracheostomy in Patients with COVID-19: Comparison and Outcomes of an Institutional Series in New York City. Ann Surg. 2021;273(3):403-9.

14. Durbin CG, Jr., Perkins MP, Moores LK. Should tracheostomy be performed as early as 72 hours in patients requiring prolonged mechanical ventilation? Respir Care. 2010;55(1):76-87.

15. Wang R, Pan C, Wang X, Xu F, Jiang S, Li M. The impact of tracheotomy timing in critically ill patients undergoing mechanical ventilation: A meta-analysis of randomized controlled clinical trials with trial sequential analysis. Heart Lung. 2019;48(1):46-54.

16. Kwak PE, Connors JR, Benedict PA, Timen MR, Wang B, Zhang Y, et al. Early Outcomes From Early Tracheostomy for Patients With COVID-19. JAMA Otolaryngol Head Neck Surg. 2021;147(3):239-44.

17. Tran K, Cimon K, Severn M, Pessoa-Silva CL, Conly J. Aerosol generating procedures and risk of transmission of acute respiratory infections to healthcare workers: a systematic review. PLoS One. 2012;7(4):e35797.

18. Wei WI, Tuen HH, Ng RW, Lam LK. Safe tracheostomy for patients with severe acute respiratory syndrome. Laryngoscope. 2003;113(10):1777-9.

19. Givi B, Schiff BA, Chinn SB, Clayburgh D, lyer NG, Jalisi S, et al. Safety Recommendations for Evaluation and Surgery of the Head and Neck During the COVID-19 Pandemic. JAMA Otolaryngology-Head \& Neck Surgery. 2020;146(6):579-84.

20. Smith D, Montagne J, Raices M, Dietrich A, Bisso IC, Las Heras M, et al. Tracheostomy in the intensive care unit: Guidelines during COVID-19 worldwide pandemic. Am J Otolaryngol. 2020;41(5):102578.

21. Sommer DD, Engels PT, Weitzel EK, Khalili S, Corsten M, Tewfik MA, et al. Recommendations from the CSO-HNS taskforce on performance of tracheotomy during the COVID-19 pandemic. J Otolaryngol Head Neck Surg. 2020;49(1):23.

22. Lamb CR, Desai NR, Angel L, Chaddha U, Sachdeva A, Sethi S, et al. Use of Tracheostomy During the COVID-19 Pandemic: American College of Chest Physicians/American Association for Bronchology 
and Interventional Pulmonology/Association of Interventional Pulmonology Program Directors Expert Panel Report. Chest. 2020;158(4):1499-514.

23. Riskjusteringsmodeller inom svensk intensivvård Svenska intensivvårdsregistret2016 [cited 2021 May 4]. 17.0:[Available from: https://www.icuregswe.org/globalassets/riktlinjer/riskjustering.pdf.

24. Safety and 30-day outcomes of tracheostomy for COVID-19: a prospective observational cohort study. Br J Anaesth. 2020;125(6):872-9.

25. Soeroto AY, Soetedjo NN, Purwiga A, Santoso P, Kulsum ID, Suryadinata H, et al. Effect of increased $\mathrm{BMI}$ and obesity on the outcome of COVID-19 adult patients: A systematic review and meta-analysis. Diabetes Metab Syndr. 2020;14(6):1897-904.

26. Lee RM, Tan GP, Puah SH, Ling LM, Choy CY, Chotirmall SH, et al. Physiological Changes During Prone Positioning in COVID-19 Acute Respiratory Distress Syndrome. Ann Acad Med Singap. 2020;49(7):509-13.

27. El Solh AA, Jaafar W. A comparative study of the complications of surgical tracheostomy in morbidly obese critically ill patients. Crit Care. 2007;11(1):R3.

28. Young D, Harrison DA, Cuthbertson BH, Rowan K. Effect of early vs late tracheostomy placement on survival in patients receiving mechanical ventilation: the TracMan randomized trial. Jama. 2013;309(20):2121-9.

29. Chorath K, Hoang A, Rajasekaran K, Moreira A. Association of Early vs Late Tracheostomy Placement With Pneumonia and Ventilator Days in Critically III Patients: A Meta-analysis. JAMA Otolaryngol Head Neck Surg. 2021.

30. Avilés-Jurado FX, Prieto-Alhambra D, González-Sánchez N, de Ossó J, Arancibia C, Rojas-Lechuga MJ, et al. Timing, Complications, and Safety of Tracheotomy in Critically III Patients With COVID-19. JAMA Otolaryngol Head Neck Surg. 2020;147(1):1-8.

31. Chao TN, Harbison SP, Braslow BM, Hutchinson CT, Rajasekaran K, Go BC, et al. Outcomes After Tracheostomy in COVID-19 Patients. Ann Surg. 2020;272(3):e181-e6.

32. McGrath BA, Brenner MJ, Warrillow SJ, Pandian V, Arora A, Cameron TS, et al. Tracheostomy in the COVID-19 era: global and multidisciplinary guidance. Lancet Respir Med. 2020;8(7):717-25.

33. Volo T, Stritoni P, Battel I, Zennaro B, Lazzari F, Bellin M, et al. Elective tracheostomy during COVID-19 outbreak: to whom, when, how? Early experience from Venice, Italy. Eur Arch Otorhinolaryngol. 2021;278(3):781-9.

34. Nishio N, Hiramatsu M, Goto Y, Shindo Y, Yamamoto T, Jingushi N, et al. Surgical strategy and optimal timing of tracheostomy in patients with COVID-19: Early experiences in Japan. Auris Nasus Larynx. 2021;48(3):518-24.

\section{Figures}




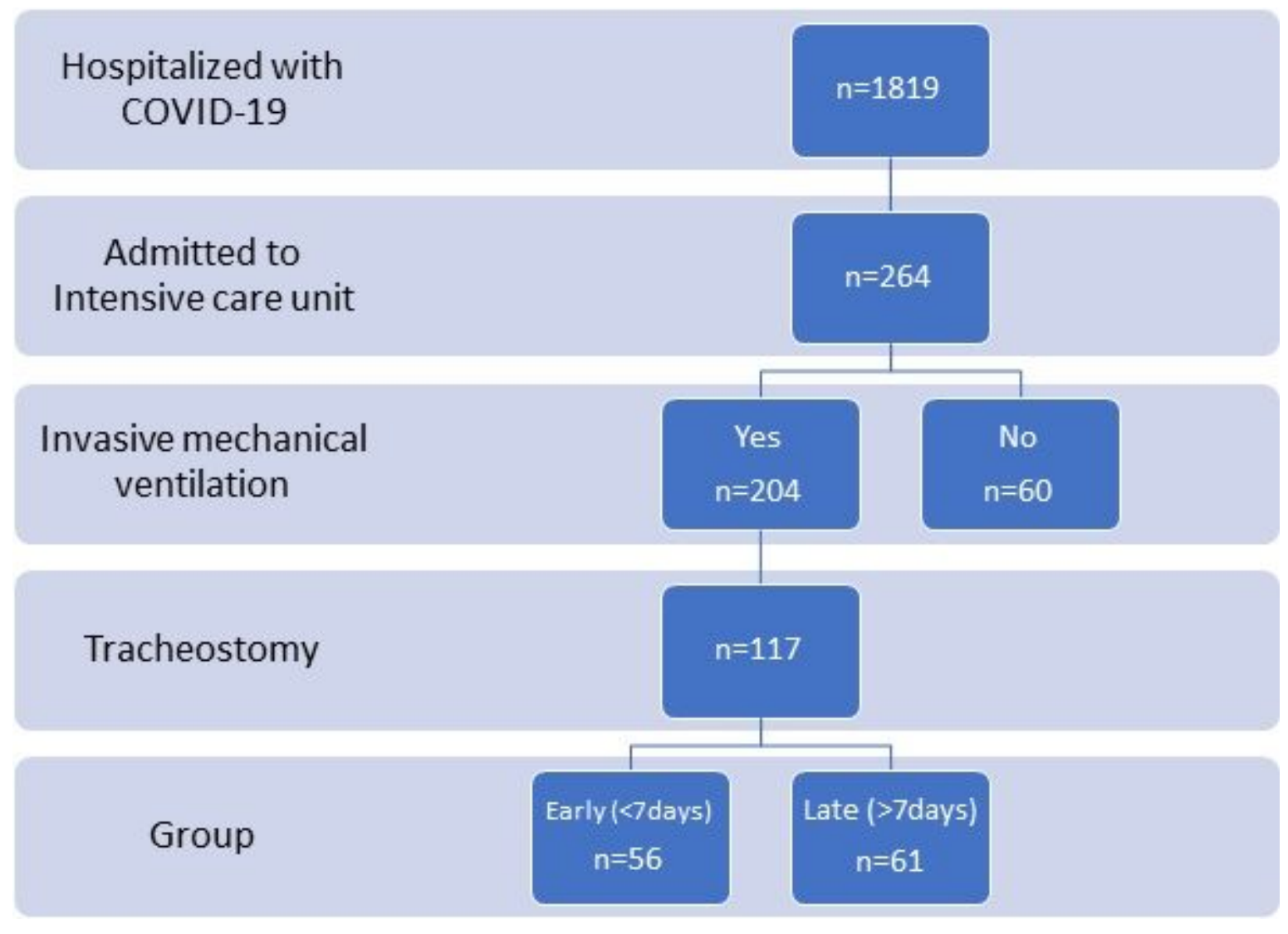

Figure 1

Patient selection flowchart 